\title{
New Solubility Test by Sliding on Ag-Pd-Cu-Au Alloys with Different Cu Contents into Different Solutions with or Without Mucin
}

\author{
Seigo Okawa, Kenji Izumi \\ Division of Biomimetics, Niigata University Graduate School of Medical and Dental Sciences \\ 2-5274, Gakkoucho-dori, Chuo-ku, Niigata, 951-8514, Japan \\ sokawa@dent.niigata-u.ac.jp
}

Corresponding Author:

\section{Seigo Okawa}

Division of Biomimetics, Niigata University Graduate School of Medical and Dental Sciences

2-5274, Gakkoucho-dori, Chuo-ku, Niigata, 951-8514, Japan

sokawa@dent.niigata-u.ac.jp

\begin{abstract}
Aims: The aims of this study was designed to analyze the released ions from Ag-Pd-Cu-Au alloys after a new sliding solubility test in different solutions with or without mucin. Materials and methods: Eluate solutions, similar to Fusayama's artificial saliva, Hanks buffer solution, and artificial saliva with or without mucin were prepared. Ag-Pd-Cu-Au casting alloys were selected by particularly examining Cu of 0-20 wt\%. The casting specimen surface was rubbed with zirconia balls in the eluate solutions using a seesaw movement for five minutes every hour for 7 days at $37^{\circ} \mathrm{C}$. The amounts of ions were detected using inductively coupled plasma mass spectroscope (ICPMS). Data were analyzed using one-way repeated analysis of variance (ANOVA) and Tukey's multiple comparisons $(\alpha=0.05)$. Results: According to the addition of mucin to the solutions, amounts of elute $\mathrm{Cu}$ ions increased in higher Cu content alloys. Similarly, the elution of Zn ions was found to be increasing, with minor exceptions, among the alloys. As for the Pd ion elution, it was interesting that the amount of Pd ions decreased, with the exception for some eluate solutions. The dissolution of In ions can be expected to progress rapidly. The behavior of the released Ag ions was complex because it differed according to either the alloy or the elute solution, or both. Conclusions: For higher Cu content alloys, amounts of elute $\mathrm{Cu}$ ions increased in the elute solutions with mucin. In contrast, amounts of other metallic ions increased and decreased or showed no change depending on the test conditions. The differences of alloy components and elute solutions might be affected by the amounts of elute ions in this sliding solubility test. Not until the elute conditions were the same did the values for ion concentrations compare with conventional reported values.
\end{abstract}

Keywords: solubility test by sliding, eluate ions, $\mathrm{Ag}-\mathrm{Pd}-\mathrm{Cu}$-Au alloys, mucin, ICPMS

\section{INTRODUCTION}

Examinations of biomaterials' mechanical, chemical, and biological features were conducted to characterize them. Corrosion tests were done to examine the release of metal ions in a solution, with emphasis on biological examination. Corrosion of an alloy is of fundamental importance to its biocompatibility because the release of elements from an alloy is nearly always necessary for adverse biologic effects to occur, such as toxicity, allergy, and mutagenicity. Therefore, corrosion has been 
studied using many methods. Many reports have described biometal characteristics [1-6].

From examination of release elements and tarnishing of biometals, experimental methods of immersion in inorganic acid solutions and an electrochemical corrosion tests were established in Japanese industrial standards (JIS) [7, 8] and the international organization for standardization (ISO) [9]. According to these standards, analyses have examined metal ions released from alloys for a fixed immersion period in an electrochemical system. Metal ion release and corrosion behavior of alloys have been evaluated. These methods are typically designated as static solubility tests. Ichinose [10] reported the amount of the release of $\mathrm{Cu}$ elements from $\mathrm{Ag}-\mathrm{Pd}-\mathrm{Cu}-\mathrm{Au}$ alloy for dental materials in a normal saline solution for a certain period. Wataha et al. [11] reported that the amount of released $\mathrm{Ag}$ and $\mathrm{Cu}$ ions from its alloy increased when the alloy was immersed in a saline-protein solution. These reports clarified the differences among amounts of the released ions for elute solutions. Therefore, different solutions can be expected to engender different corrosion behaviors of $\mathrm{Ag}-\mathrm{Pd}-\mathrm{Cu}-\mathrm{Au}$ alloy. Moreover, some interesting reports have described that amounts of released metal ions increased [12, 13] or decreased $[14,15]$ or remained unchanged [16] using the eluted solutions, including amino acid or proteins, for which the behavior of released ions depended on the heat treatment of alloys [10].

In contrast, a dynamic solubility test was proposed by Miura et al. [17]. By this test, a dental alloy was slid on a surface of packed ceramic balls to simulate the abrasion of a dental prosthesis in an oral cavity through eating and occlusion. In addition, Akagi et al. [18] studied the effect of the release of metal ions on cytotoxicity under a dynamic solubility test. According to this report, the dynamic extraction solubility test was effective for the formation of a model of a new cytotoxicity test. The behavior of released metal ions depends on the alloy composition, immersion liquid component, and the test environment. However, both the dynamic solubility test and the dynamic extraction solubility test used conditions of contact between the specimen surface and the ball. The specimen weight and the sliding speed are expected to affect the amounts of the released metal ions. Under some dynamic solubility testing conditions, metal debris might be created, in contrast to the traditional metal solubility test.

Therefore to evaluate the amounts of released metal ions from dental alloys under similar conditions of the oral cavity, a method of zirconia balls sliding on the metal surface was applied for the corrosion tests described in this report. This method presents several benefits: it has no effect on the specimen weight, sliding speed, or visible debris. Hereinafter, these tests are designated as sliding solubility tests.

This study was designed to analyze the released ions using inductively coupled plasma mass spectroscopy (ICPMS) after the sliding solubility test of Ag-Pd-Cu-Au alloys with different $\mathrm{Cu}$ content. It was also conducted to investigate the effects of mucin in elute solutions.

\section{MATERIALS AND METHODS}

\section{Preparation of Specimens}

Major types of $\mathrm{Ag}-\mathrm{Pd}-\mathrm{Cu}-\mathrm{Au}$ casting alloys available for clinical use were selected by specifically examining $\mathrm{Cu}$ of $0-20 \mathrm{wt} \%$. The alloys were Castwell M.C. (12\% Gold) (GC Corporation, Tokyo, Japan), Superloy I (Morita Corpration, Tokyo, Japan) and Tsutsumida Golden Soft I (Tsutsumida kikinzoku kougyou Ltd., Hiroshima, Japan). The codes were given as, "CW" for Castwell M.C.(12\% Gold), "SA" for Superloy I, "GS" for Tsutsumida Golden Soft I. Table 1 presents the weight percentage composition of the alloys presented by the manufacturers. Alloys were cast into rectangular specimens $10 \times 20 \times 1.6 \mathrm{~mm}^{3}$ according to conventional casting methods. Specimens $(n=3)$ were finally polished with \#1000 SiC waterproof paper under tap water using a polishing unit(TegraPoll 11, Struers ApS, Ballerup, Denmark). Then they were cleaned ultrasonically in methanol and distilled water. 
New Solubility Test by Sliding on Ag-Pd-Cu-Au Alloys with Different Cu Contents into Different Solutions with or Without Mucin

Table 1. Weight percentage compositions of the alloys reported by the manufacturers

\begin{tabular}{|c|c|c|c|c|c|c|c|c|}
\hline \multirow{2}{*}{ Code } & \multicolumn{7}{c|}{ Elements } \\
\cline { 2 - 9 } & $\mathrm{Au}$ & $\mathrm{Pd}$ & $\mathrm{Ag}$ & $\mathrm{Cu}$ & Zn+Ir+In & $\mathrm{Zn+In+Sn}$ & $\mathrm{In}$ & $\mathrm{Ir}$ \\
\hline GS & 12 & 20.2 & 48.7 & 0 & & & 17 & 2.05 \\
\hline SA & 12 & 20 & 52.5 & 13 & & 2.5 & & \\
\hline CW & 12 & 20 & 46 & 20 & 2 & & & wt\% \\
\hline GS: Tsutsumida Golden Soft I, SA: Superloy I & & \\
CW: Castwell M.C.(12\% Gold), & & & \\
\hline
\end{tabular}

\section{Eluate Solutions}

Eluate solutions for the sliding solubility test were the following: (1) Artificial saliva similar to Fusayama's artificial saliva $(A+M)$ was prepared based on the literature [19]; (2) Hanks buffer solution (H-M, H8264; Sigma-Aldrich Corp., St. Louis, USA); and (3) Artificial saliva (S-M, Saliveht; Teijin Pharma Ltd., Tokyo, Japan). Mucin (Wako Pure Chemical Industries Ltd., Osaka, Japan), a protein derived from pig stomach cells, was added to the H-M and S-M. Then H+M and $\mathrm{S}+\mathrm{M}$ were prepared as eluate solutions including mucin. Furthermore, artificial saliva without mucin (A-M) was prepared. Table 2 presents the compositions of the eluate solutions used for this study.

Table 2. Compositions of eluate solutions used for this study

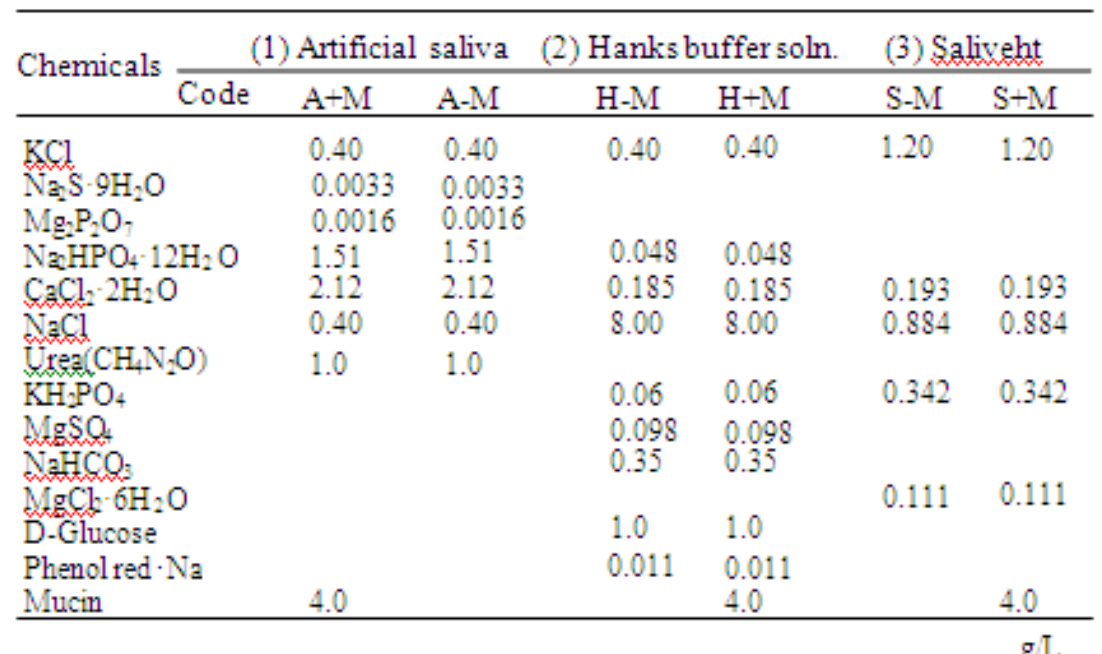

\section{Sliding Solubility Test}

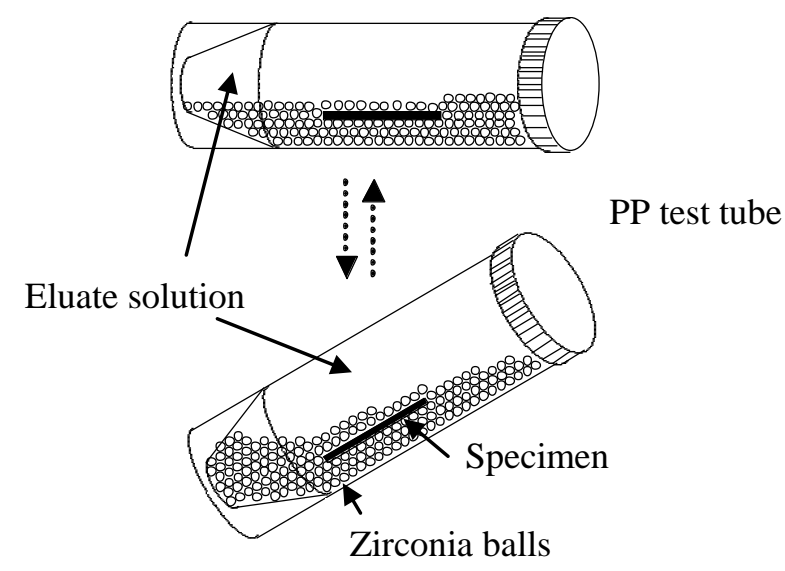

Figure 1. Outline of a sliding solubility test. 
Into a PP test tube, $50 \mathrm{~mL}$ of the eluate solution and $30 \mathrm{~g}$ of 2-mm-diameter zirconia balls were put along with the cast specimen, which had been cleaned ultrasonically in ethanol. After screwing on the cap of the PP test tube, it was set on a bed of a laboratory shaker (RM-300; AS One Corp., Tokyo, Japan). When the laboratory shaker bed was seesawed at angles of 0-45 deg, the zirconia balls slid up and down. Then the specimen surface was rubbed with the balls in the test tube. A schematic of the sliding solubility test is presented in Fig. 1. The laboratory shaker was operated for five minutes every hour. This test was conducted for 7 days at $37^{\circ} \mathrm{C}$ in a dry bath.

\section{Analysis of metal ions released from the alloys}

After the sliding solubility test, $2 \mathrm{~mL}$ of the eluate solution and ultrapure $\mathrm{HNO}_{3}$ (Tama Chemicals Co. Ltd., Tokyo, Japan) were added to a Teflon pressure-tight case. Then the case was left in a thermostatic chamber at $120^{\circ} \mathrm{C}$ for $2 \mathrm{hr}$. After cooling, the treated solution was used for analysis of metal ions. An inductively coupled plasma mass spectroscope (ICPMS, ICPM-8500; Shimadzu Corp., Kyoto, Japan) was used to assess the released metal ions of each alloy. Table 3 presents analytical conditions used for ICPMS in this study. This sensitive technique was used to ascertain which ions were primarily released from the alloy under the sliding solubility test.

Table 3. Analytical conditions using ICPMS in this study

\begin{tabular}{|l|c|}
\hline Power of high-frequency wave & $1.2 \mathrm{~kW}$ \\
\hline Sampling depth & $3.5 \mathrm{~mm}$ \\
\hline Cloolant gas & $7.0 \mathrm{~L} / \mathrm{min}$ \\
\hline Plasma gas & $1.5 \mathrm{~L} / \mathrm{min}$ \\
\hline Carrier gas & $0.60 \mathrm{~L} / \mathrm{min}$ \\
\hline Quantitative profile points & 11 \\
\hline Quantitative integral points & 1 \\
\hline Number of cycles of integral & 20 \\
\hline
\end{tabular}

\section{Statistical analysis}

Statistically significant differences between groups were assessed using one-way analysis of variance and Tukey multiple comparison intervals $(\alpha=0.05)$.

\section{RESUlTS AND DisCUSSION}

Figures 2(a)-2(e) show amounts of released $\mathrm{Cu}, \mathrm{Zn}, \mathrm{Pd}, \mathrm{In}$, and Ag ions from Ag-Pd-Cu-Au dental casting alloys into the elute solutions without or with mucin. The SD was low for all specimens. There were no data of $\mathrm{Cu}$ for $\mathrm{GS}$ because this alloy contains no $\mathrm{Cu}$. The maximum released $\mathrm{Cu}$ was approximately $510 \mathrm{ppb}$ in the case of $\mathrm{CW}$ into $\mathrm{H}+\mathrm{M}$. The amount was found to be 1.3-6.0 times with mucin for $\mathrm{SA}$ and $\mathrm{CW}$ alloys compared to the amount without mucin. For the elute solutions $\mathrm{A}+\mathrm{M}$, $\mathrm{H}+\mathrm{M}$, and $\mathrm{S}+\mathrm{M}$, the released $\mathrm{Cu}$ ions of $\mathrm{CW}$ were, respectively, around 1.4, 5.5, and 2 times those found in A-M, H-M, and S-M. Similarly, the concentrations for SA were, respectively, around 3.6, 3, and 1.6 times those found in A-M, H-M, and S-M. In general, although not always, greater amounts of released ions were evident in solutions with mucin than in solutions without mucin. The concentration of the released $\mathrm{Cu}$ ions increased depending on the alloy with higher $\mathrm{Cu}$ content. Wright et al. [20] demonstrated that amount of released $\mathrm{Cu}$ ion was related to the $\mathrm{Cu}$ content of alloy. The amounts of metal ions increased in the solution with mucin. One reason was that mucin absorbed the released metal ions, especially $\mathrm{Cu}$ and $\mathrm{Zn}$ ions [17]. The concentrations of metal ions on the alloy surface decreased so that the metal ions would be released from it. Moreover, a new fresh alloy surface would 
appear according to the sliding solubility test. Then corrosion would progress. However, in a solution without mucin, the released metal ions might form a hydroxide and a chloride. The alloy surface was covered with these compounds to delay the release of metal ions. By dynamic and static tests, the chemical compounds would be partially removed from the surface. The sliding solubility test, however, was not sufficiently strong. The concentration of the released metal ions might be low, which is compatible with the results. Another reason is given below. The prepared artificial saliva $(\mathrm{A}+\mathrm{M})$ contained urea. For this elute solution, the $\mathrm{Cu}$ ion concentration increased clearly. Urea reacted with released $\mathrm{Cu}$ ion to form $\mathrm{CuCl}_{2} \cdot 2\left(\mathrm{CH}_{4} \mathrm{H}_{2} \mathrm{NO}\right)$ [4]. Action of masking by this compound can be expected to work on the alloy surface. No effect of the masking work was found in this study because of the sliding action. However, an oxygen concentration cell on the alloy surface would be formed. Consequently, the elution of $\mathrm{Cu}$ ions would increase.

Similarly, the elution of $\mathrm{Zn}$ ions was found to be increasing, with minor exceptions, among the alloys for the elute solution with mucin. The maximum concentration of $\mathrm{Zn}$ was approximately $650 \mathrm{ppb}$ in the case of $\mathrm{CW}$ into $\mathrm{A}+\mathrm{M}$. The concentration was approximately nine times that into A-M. Some interaction between mucin and chemical components might advance the state of corrosion. In addition, surface morphology of the specimen might affect the release of metal ions. In other words, a casting defect might cause ion release.

As for the Pd ion elution, it was interesting that the amount of Pd ions decreased for the treated solution with mucin, with the exception for SA into A+M. The maximum Pd concentration was about $11 \mathrm{ppb}$. Mucin might be unaffected by the release of Pd ions.

Results show that the release of In ions from GS increased. The concentration for GS into H+M was approximately $50 \mathrm{ppb}$. However, it was interesting that few In ions were released from the other alloys, SA and CW, into the elute solutions. Consequently, according to the addition of mucin to the solutions, the dissolution of In ions can be expected to progress rapidly. This dissolution behavior would depend, not surprisingly, on the alloy component. Therefore, the differences of the components of the alloy can be expected to cause different corrosion behavior from those associated with SA and CW alloys.

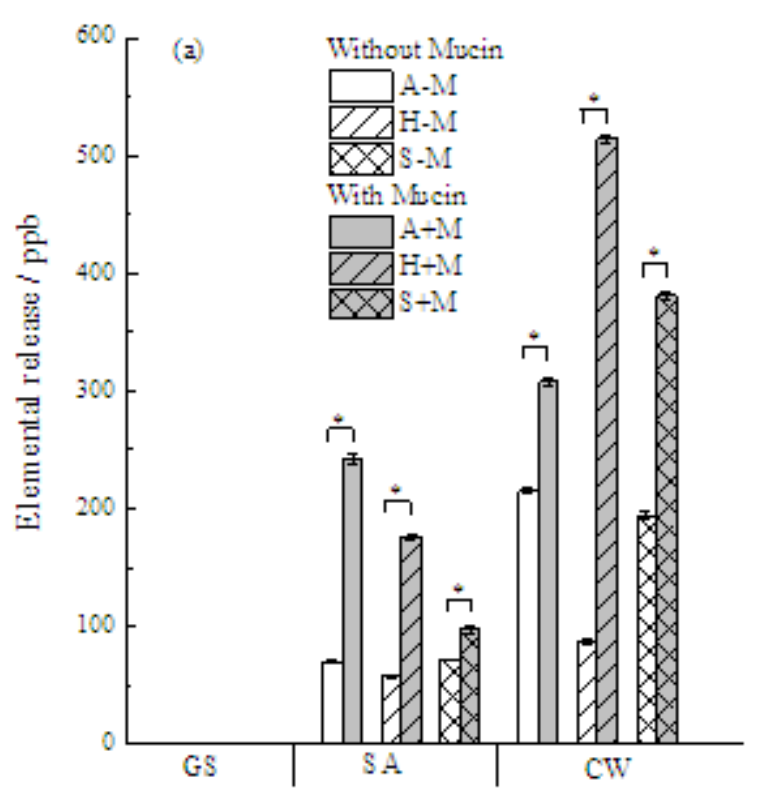

Figure 2. Elemental release (a) Cu ions. 


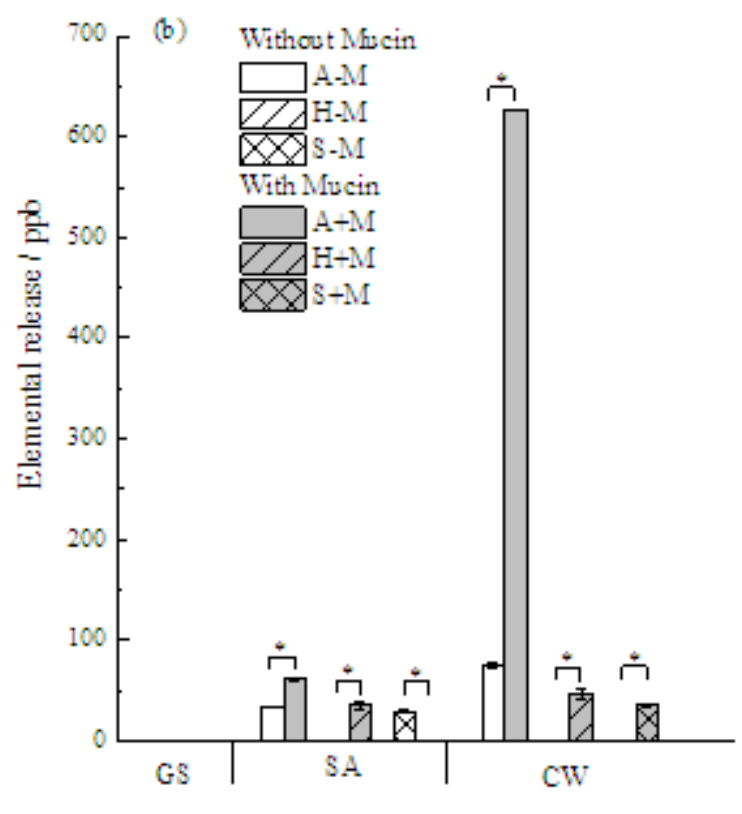

Figure 2 (b) Zn ions

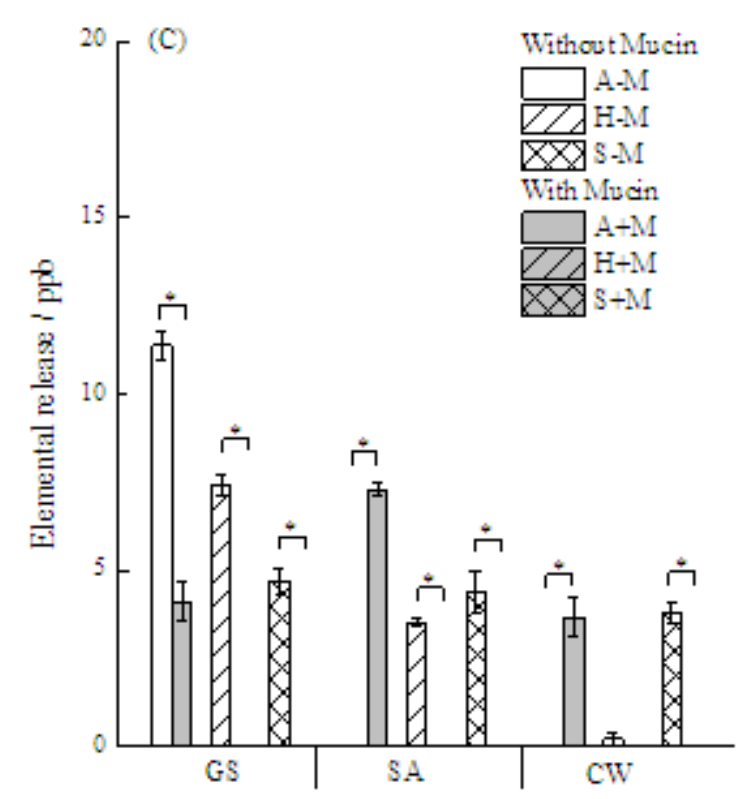

Figure 2 (c) Pd ions

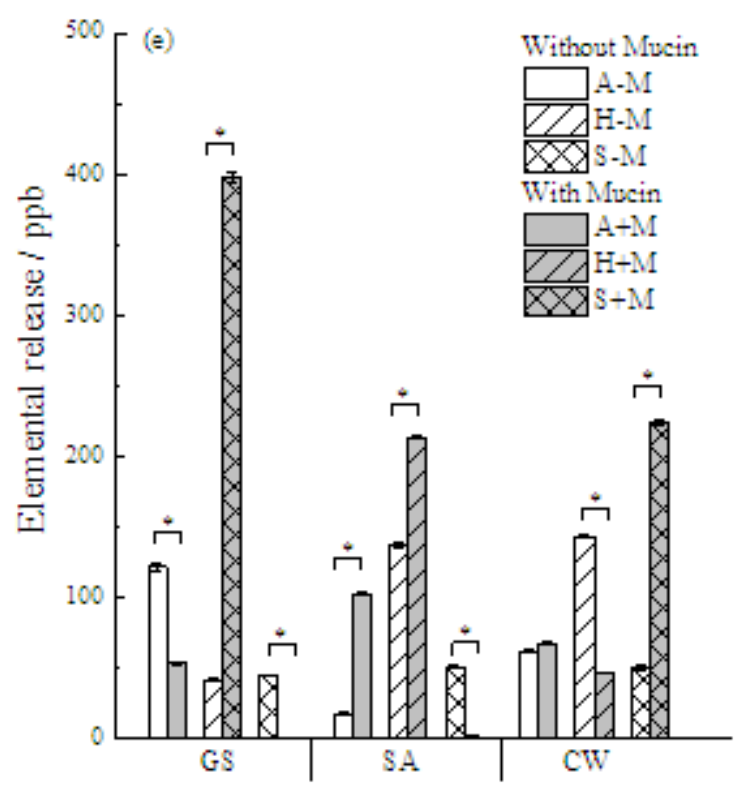

Figure 2 (d) In ions

Figure 2 (e) Ag ions

The behavior of the released Ag ions was complex because it differed according to either the alloy or the elute solution, or both. The concentration of Ag ions of $\mathrm{CW}$ into S+M was about 4.6 times that into S-M. It was approximately 9.5 times into $\mathrm{H}+\mathrm{M}$ compared to that into $\mathrm{H}-\mathrm{M}$ in the case of GS. Moreover, for SA, it was approximately 1.6 times into $\mathrm{H}+\mathrm{M}$ compared to that into H-M. It was interesting that no elution of $\mathrm{Ag}$ ions occurred in cases of GS and SA into S+M. The concentration of $\mathrm{Ag}$ ions in the case of $\mathrm{CW}$ into $\mathrm{H}+\mathrm{M}$ was approximately one-third. In the case of GS into $\mathrm{A}+\mathrm{M}$, it was approximately half that of the treated solution without mucin. However, in the case of $\mathrm{CW}$ into $\mathrm{A}+\mathrm{M}$, 
the concentration was as much as that for the solution without mucin. In the case of GS and SA into $\mathrm{S}+\mathrm{M}$, the elution of $\mathrm{Ag}$ ions was only slightly detected. Consequently, two function effects were found to occur in the presence of mucin. The corrosion would be prevented or would be promoted depending on the treated solutions. In addition, the difference in the behavior of the released ions would cause different corrosion behavior using the component of the solutions and the environment. Absorption of dental plaque and proteins on the alloy surface can cause the formation of oxygen concentration cells on it [21]. In this study, that stands as one reason explaining why the amounts of metallic ions were released from the alloy.

\section{Conclusions}

To investigate the effects of different solutions with or without mucin on corrosion of Ag-Pd-Cu-Au alloys, a new sliding solubility test was conducted. The amounts of metallic ions were detected using ICPMS. For higher $\mathrm{Cu}$ content alloys, amounts of elute $\mathrm{Cu}$ ions increased in the elute solutions with mucin. In contrast, amounts of other metallic ions increased and decreased or showed no change depending on the test conditions. The differences of alloy components and elute solutions might be affected by the amounts of elute ions in this sliding solubility test. Not until the elute conditions were the same did the elute ion concentration compare with conventionally reported concentrations.

\section{ACKNOWLEDGMENTS}

We would like to thank our former technical official Honma Hiro for her invaluable ICPMS work.

\section{Conflicts of interest}

There are not conflicts of interest.

\section{REFERENCES}

[1] Caputo A, Zucchi F, In: Barbucci R, editor. Integrated biomaterials science. Dordrecht, Kluwer Academic Plenum, 2002, pp. 297-324.

[2] Upadhyay D, Panchal MA, Dubey RS, Srivastava VK, Corrosion of alloys used in dentistry: A review, Mater Sci Eng. A432, 1-11 (2006).

[3] Wataha JC, Biocompatibility of dental casting alloys: a review, J Prosthet Dent. 83, 223-234 (2000).

[4] Matsuda K, Uno Y, Sakai H, Inoue H, Kimura K, Shimokobe H, In vitro and in vivo study on corrosion behavior of a Ag-Pd-Cu-Au alloy, J J Conserv Dent. 29(1), 260-264 (1986).

[5] Nieml L, Minni E, Ivaska A, An electrochemical and multispectroscopic study of corrosion of Ag-Pd-Cu-Au alloys, J Dent Res. 65(6), 888-891 (1986).

[6] Geurtsen W, Biocompatibility of dental casting alloys, Crit Rev Oral Bio Med. 13(1), 71-84 (2002).

[7] JIS T6106 Dental casting gold-silver-palladium alloys

[8] JIS T 6002 Dental metallic materials - Corrosion test methods

[9] ISO 10271 Corrosion test methods for metallic materials

[10] Ichinose S, Corrosion behavior of dental Ag-Pd-Cu-Au Alloy in various solutions, J J Dent Mater. 11(1), 149-168 (1992).

[11] Wataha JC, Nelson SK, Lockwood PE, Elemental release from dental casting alloys into biological media with and without protein, Dental Materials. 17, 409-414 (2001).

[12] Brown SA, Merritt K, Electrochemical corrosion in saline and serum, J Biomed Mater Res. 14(2), 173-175 (1980). 
[13] Williams DF, Clark GCF, The corrosion of pure cobalt in physiological media, J Mater Sci. 17(6), 1675-1682 (1982).

[14] Brown SA, Merritt K, Fretting corrosion in saline and serum, J Biomed Mater Res. 15, 479-488 (1981).

[15] Johansson BI, In vitro study of galvanic currents between amalgam and gold alloy electrode in saliva and in saline solutions, Eur J Oral Sci. 94(6), 562-568 (1986).

[16] Hero H, Hultquist G, Oden A, Selective dissolution of $\mathrm{AuCu}$ and $\mathrm{AuAg}$ alloys in vitro and vivo, Biomater. 6(6), 393-395 (1985).

[17] Miura Y, Takeda, Effects of extraction condition on cytotoxicity testing of metallic biomaterials, J J Dent Mater. 14(2), 253-264 (1995).

[18] Akagi H, Takeda S, Nakamura M, Dissolution and cytotoxicity of gold-silver-palladium alloy, J J Dent Mater. 19(2), 179-186 (2000).

[19] Fusayam T, Katayori T, Nomoto S, Corrosion of gold and amalgam placed in contact mutually, J Dent Res. 42(5), 1183-1197 (1963).

[20] Wright DC, Gallant RF, Spangberg L, Correlation of corrosion behavior and cytotoxicity in AuCu-Ag ternary alloys, J Biomed Mater Res. 16(4), 509-517 (1982).

[21] Sakane K, Takeda S, Nakamura M, In vitro corrosion of precious alloys in the presence of cells, J Osaka Odontol Soc. 64(3), 253-260 (2001). 